\title{
Las IDE 3D: necesidad, desarrollo y líneas de investigación
}

\author{
Javier Valencia* \\ Ángel Luis Muñoz ${ }^{* *}$
}

Recibido el 2 de febrero de 2018; aceptado el 6 de abril 2018

\section{Resumen}

Este artículo es un resumen de la tesis doctoral desarrollada sobre la situación actual de las IDE con respecto a la tercera dimensión, abordando líneas de investigación a futuro y analizando las necesidades y debilidades que se presentan. Tras profundizar en los conocimientos sobre IDE y, tras comprobar tanto la necesidad como la convergencia de diversas ramas técnico-científicas en materia de gestión de información geográfica (IG) tridimensional, se decide, a través de este trabajo desarrollar un proyecto de investigación centrado en las IDE 3D.

Siguiendo un enfoque cualitativo en el desarrollo de la metodología de investigación, se hace patente la importancia de lo que supone la tercera dimensión espacial para el desempeño de las actividades cotidianas del ser humano en nuestros tiempos. Finalmente, se definen los elementos necesarios para poder implementar una IDE tridimensional, teniendo en cuenta todos los parámetros de desarrollo, tanto técnicos como legales, necesarios para cumplir con los actuales marcos tecnológicos, normativos, jerárquicos y legales.

A través del trabajo desarrollado, se ha constatado que, por lo general, la adaptación de la tercera dimensión por parte de las IDE está en fase germinal, con muchas posibilidades de avance y aporte de contribuciones científicas y tecnológicas. Este documento intenta ser una de esas contribuciones.

Palabras clave: IDE, 3D, CityGML, BIM, WebGL.

* LYRA ingeniería \& consultoría, España, correo electrónico: javi.valencia.m@gmail.com

** Universidad de Salamanca, España, correo electrónico: almuni@usal.es 


\section{Resumo}

Este artigo é um resumo da tese de doutorado desenvolvida sobre a situação atual das IDE com respeito a terceira dimensão, abordando linhas de investigação futuras e analisando as necessidades e deficiências que se apresentam. Depois de aprofundar os conhecimentos sobre IDE e de comprovar tanto a necessidade como a convergência de diversas vertentes técnico-científicas em matéria de gestão de informação geográfica (IG) tridimensional, se decide, através deste trabalho desenvolver um projeto de investigação centrado nas IDE 3D.

Seguindo um enfoque qualitativo no desenvolvimento da metodologia de investigação, se faz latente a importância do que implica a terceira dimensão espacial para o desempenho das atividades cotidianas nos nossos tempos. Finalmente, se definem os elementos para poder implementar uma IDE tridimensional, tendo em conta todos los parâmetros de desenvolvimento, tanto técnicos como legais, necessários para cumprir com os atuais marcos tecnológicos, normativos, hierárquicos e legais.

Através do trabalho desenvolvido, se constatou que, em geral, a adaptação da terceira dimensão por parte das IDE está em fase germinal, com muitas possibilidades de avanços e aportes de contribuições científicas e tecnológicas. Este documento intenta ser uma dessas contribuições.

Palavras chave: IDE, 3D, CityGML, BIM, WebGL

\section{Abstract}

This article is a summary of the doctoral thesis developed about the current situation of SDI with respect to the third dimension, addressing future research lines and analysing the needs and weaknesses showed. After deepening knowledge about SDI and, after checking both the need and the convergence of various technical-scientific branches in terms of three-dimensional geographic information (GI) management, it is decided, through this work, to develop a research project focused on 3D SDI.

Following a qualitative approach in the development of the research methodology, the importance of what the third spatial dimension supposes for the performance of the daily activities of the human being in our times becomes clear. Finally, the necessary elements to be able to implement a three-dimensional SDI are defined, taking into account all the development parameters, both technical and legal, necessary to comply with the current technological, regulatory, hierarchical and legal frameworks.

Through the work developed, it has been found that, in general, the adaptation of the third dimension by the SDI is in germinal phase, with many possibilities for advancement and contribution of scientific and technological contributions. This document tries to be one of those contributions.

Key words: IDE, 3D, CityGML, BIM, WebGL. 


\section{La tercera dimensión espacial. Una necesidad para las IDE}

Desde principios de este siglo XXI, se comenzó a vislumbrar la necesidad de trabajar con la tercera dimensión en aplicaciones relacionadas con la bioingeniería y la medicina (Montilla, G.; Bosnjak, A. y Villegas, H., 2002). También se apreciaba esta necesidad en grandes industrias como la cinematográfica y la pujante industria de los juegos (Ahearn, Luke, 2005), en la que no se concibe la fase de diseño sin la recreación de modelos tridimensionales complejos.

Del mismo modo, en arquitectura e ingeniería civil, la tercera dimensión se consolida como información vital para el desarrollo y avance de estas disciplinas tanto en tareas de diseño y proyecto (Monedero, J., 2001), como en labores de ingeniería inversa (Lafont Morgado, P., 1999). De este modo, se constata cómo alrededor de estas disciplinas surgen nuevos conceptos y nuevos campos de uso de este tipo de información: Building Information Models (BIM), recreación tridimensional forense, modelización de sistemas urbanos complejos, etc. La tercera dimensión espacial se ha convertido en una auténtica necesidad para muchas aplicaciones técnico-científicas.

En el mundo de las geotecnologías y, más concretamente en el ámbito de los Sistemas de Información Geográficos (SIG o GIS), se ha pasado de utilizar la tercera dimensión como un atributo o dato adicional que acompaña a un elemento geográfico (Figura 1), a ser un dato esencial para la realización de análisis espaciales (Scianna y Ammoscato, 2010).
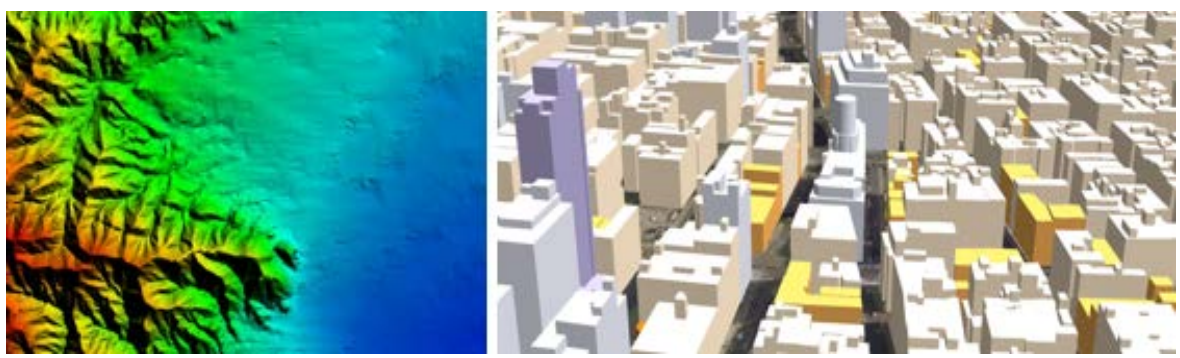

Figura 1. Simbolización de la tercera dimensión en un sistema 2D (izquierda) vs. análisis espacial tridimensional con tecnología WebGL (derecha). Elaboración propia mediante Global Mapper y Cesium. 
Al tiempo que la tercera dimensión ha ido creciendo en importancia impulsada por el desarrollo de aplicaciones que se sirven de ella, de manera paralela, la obtención de información tridimensional ha dejado de ser un cuello de botella inducido por el esfuerzo tecnológico y económico que conllevaba hace escasos años su captura y procesamiento. Los medios de adquisición y tratamiento que se utilizan en la actualidad han reducido sus costes y tamaño, pasando de prototipos experimentales no comerciales de la última década del siglo $\mathrm{xX}$ a asequibles productos comerciales en la primera década del siglo XXI.

En nuestro "mundo real" se han establecido cuatro dimensiones que para nuestro raciocinio son claras: tres dimensiones espaciales y una dimensión temporal. Si entendemos la cartografía como una modelización de la realidad y, teniendo en cuenta lo anterior, desde el punto de vista teórico, prescindir de una dimensión espacial disminuye objetivamente la calidad del modelo (Varela García et al., 2007). Debemos tener en cuenta, además, la importancia de la tercera dimensión espacial desde el punto de vista práctico. A día de hoy son muchas las aplicaciones, que por necesidad utilizan esta dimensión espacial (de la Calle Alonso et al., 2010).

En la actualidad cualquier computadora de gama media es capaz de trabajar sin ningún problema con datos tridimensionales, renderizándolos y manipulándolos con soltura. Del mismo modo disponemos de distintos lenguajes estandarizados para el tratamiento de información 3D como VRML, Java3D y X3D (Jiménez Macías et al., 2005).

Y si la aparición de las IDE ha supuesto una auténtica revolución en la gestión, uso y difusión de la IG, sin embargo, ante la ausencia de la tercera dimensión espacial en los desarrollos y servicios IDE más comunes, están surgiendo dos problemáticas bien diferenciadas que afectan a los usuarios potenciales de las mismas. Estas dos problemáticas, que se detallarán más adelante, están dejando patente la necesidad de desarrollar todos los elementos necesarios para constituir IDE 3D y, además, se están convirtiendo en auténticos motores de desarrollo de este tipo de tecnologías, como se comprobará a continuación.

El primero de los problemas a los que se hacía referencia surge cuando algún grupo potencial de usuarios de IDE desecha esta tecnología al no resolver sus necesidades en lo que a la tercera dimensión se refiere. Un ejemplo de esto lo tenemos en la arquitectura, que desde hace tiempo viene teorizando y desarrollando los llamados modelos de información de construcción (Building Information Modeling $\mathrm{BIM}$ ), que generan y gestionan la geometría de uno o varios edificios, las relaciones espaciales entre sus elementos, la IG, así como las cantidades y las propiedades de los componentes del edificio.

El segundo problema al que se hacía mención surge por parte de aquellos usuarios de IDE, que por sus actuales necesidades, demandan de estos avances para la resolución de problemas asociados a la gestión de la tercera dimensión y que las 
IDE, hoy por hoy, no están consiguiendo satisfacer. Dentro de los ejemplos de la segunda tipología de problemas, podríamos citar el Catastro. Prácticamente desde que se inició el desarrollo de los servicios WMS y WFS, el Catastro de nuestro país y el de otras muchas naciones utilizaron dichos servicios para difundir su información. De esta manera, cualquier usuario mediante un cliente pesado, o incluso ligero, podía tener información de cualquier parcela, en cualquier momento. Sin embargo, a la hora de mostrar esta información el resultado era mediante un modelo bidimensional en el que cualquier alusión a la tercera dimensión era un mero número, que en el caso de nuestro país era un número romano (Virgós Soriano y Olivares García, 2008). Esta representación dista mucho de ser efectiva para muchas aplicaciones, por lo que al día de hoy se está procediendo a la revisión de la forma de gestionar, modelizar y publicar la información del Catastro incorporando la tercera dimensión.

Las anteriores experiencias son solo dos ejemplos de por qué las IDE, o no han podido dar solución a una determinada necesidad, o están generando tensiones para intentar resolver ciertas situaciones que se plantean en cuanto a la incorporación y gestión de la tercera dimensión.

Otro caso son las necesidades de representación del territorio, lo más realista posible que ya prometen tecnologías como las BIG Data. Y como ejemplo específico de estas últimas, podríamos citar las "Smart Cities" o ciudades inteligentes, con las que se dota de inteligencia a las infraestructuras, la información y los servicios públicos, al tiempo que se potencia la colaboración entre los diferentes agentes implicados en el entorno municipal.

Debido a la gran cantidad de datos generados desde múltiples fuentes y con diferentes estructuras, así como a las complejas necesidades de gestión e interacción, es en las IDE donde deberían encontrar respuesta las Smart Cities a la gestión de la IG y de las infraestructuras municipales.

Como puede extraerse de lo ya comentado, la tercera dimensión es toda una necesidad a la hora de contemplar las IDE como una herramienta horizontal de almacenamiento, gestión y difusión de la IG, que ya ha pasado de ser bidimensional a tridimensional.

\section{Orígenes y estado del arte de las IDE 3D}

Los orígenes del término IDE 3D se podrían establecer en el año 2008 en una publicación de Jens Basanow, Pascal Neis, Steffen Neubauer, Arne Schilling y Alexander Zipf, titulado "Towards 3D Spatial Data Infrastructures (3D-SDI) based on open standards - experiences, results and future issues" (Basanow et al., 2008). La idea fundamental del trabajo era mostrar nuevas tecnologías propuestas para la extensión en la tercera dimensión, sobre la base de la IDE de Heidelberg. 
Ya se hablaba de Web3D, de OGC (W3DS), servicio que distribuía los datos en 3D, de CityGML. Se podría decir que es el primer documento que, desde un punto de vista integrador, trata de plasmar los elementos de una IDE 3D conforme a los desarrollos de la época.

Tras esta publicación, que se integró ese mismo año en el libro "Advances in 3D geoinformation systems" (van Oosterom et al., 2008), fueron surgiendo varias e interesantes publicaciones, pero no con un punto de vista tan integrador como el de Basanow.

Sin embargo, mucho antes de esta publicación ya había organizaciones que estaban haciendo grandes desarrollos en el ámbito de la información tridimensional. Una de estas organizaciones es el Web3D Consortium, fundado en 1997. Se trata de una organización internacional, sin ánimo de lucro, financiada por los miembros (empresas privadas, universidades, gobiernos, etc.). Se encargan fundamentalmente del desarrollo de estándares para la publicación de gráficos 3D en Internet. Desarrollaron un estándar, X3D (Extensible 3D), que surgió tras VRML. X3D es un estándar abierto, extensible e interoperable (multiplataforma). X3D, como veremos más adelante, cobra gran importancia a la hora de publicar IG en Internet, ya que se constituye como una de las plataformas más utilizadas para este objetivo.

Otro punto importante de cara al desarrollo de las IDE 3D, sobre todo en lo referente a normas, lenguajes y estándares es la creación del Grupo de Trabajo sobre Gestión de la Información 3D de la OGC (The OGC’s 3D Information Management Working Group), en el año 2005.

Dicho grupo de trabajo está facilitando la definición y desarrollo de estándares que permitan soluciones de manejo y visualización de la IG tridimensional. El foco sobre el que centran sus investigaciones es el establecimiento de un marco de interoperabilidad de IG tridimensional debido a la gran variedad de productos, información y servicios existentes en la actualidad. Este trabajo es de interés para la comunidad geoespacial en el sentido de que existe una creciente necesidad de tecnologías e información para interoperar entre distintas ramas técnicocientíficas que, en la actualidad, manejan una gran cantidad de servicios y formatos de una manera dispersa. Para esto se creó el grupo, para identificar y actuar sobre las oportunidades de mejorar la interoperabilidad de datos geoespaciales tridimensionales y servicios relacionados con los anteriores.

Este grupo se está convirtiendo en un foco importante para el desarrollo de los estándares necesarios para una IDE 3D. En él participan desde los más importantes proveedores de software CAD y SIG, pasando por agencias administrativas (como la Agencia Nacional de Inteligencia Geoespacial y el Departamento de Seguridad Nacional) hasta gobiernos europeos. 
Uno de los principales hitos desarrollados ha sido la adopción de CityGML como estándar OGC (originalmente, la organización alemana de Renania del NorteWestfalia sig3D desarrolló CityGML) y su impulso, ya que proporciona un modelo estándar para describir objetos 3D con respecto a su geometría, topología, semántica y apariencia.

CityGML también proporciona una forma estándar de integrar la ubicación interior/exterior, es decir, una forma estándar de integrar la ubicación del edificio en las coordenadas globales con los detalles del edificio en coordenadas relativas de los sistemas CAD. Es, por tanto, una puerta abierta a la integración de las IDE 3D con los sistemas BIM.

Por todo lo anterior, CityGML está siendo ampliamente implementado en productos de software y servicios online. Es el estándar para la IG 3D en Holanda (potencia mundial en este tipo de modelos).

También tuvo gran repercusión la adopción de KML como estándar en el año 2008. Suponía adoptar como estándar un lenguaje que ya tenía una gran cantidad de usuarios y desarrollos relacionados con él, gracias sobre todo por el archiconocido Google Earth, plataforma original sobre la que se visualizaban este tipo de ficheros. Suponía la “democratización” de estructuras 3D más complejas en el mundo de la IG.

Ese mismo año se produce otro hito importante que tendrá fuertes repercusiones a la hora de poder hablar de IDE 3D (Basanow et al., 2008), Oracle introduce su 3D Spatial Engine en la versión 11g de su gestor de BB.DD. Desde ese momento se ha ido mejorando y desarrollando este motor para almacenar y gestionar información geométrica tridimensional, con su potencial uso, como no podía ser de otra forma, por parte de la comunidad geoespacial. Sobre este aspecto destacan las contribuciones de Peter van Oosterom, del que ya se ha hablado con anterioridad y que participó directamente en este trabajo.

De una manera algo más modesta, pero no menos importante y también relacionada con la anterior, cabe destacar la contribución que la Fundación OSGeo ha ido desarrollando, sobre todo con su proyecto PostGIS. PostGIS es una extensión del gestor de BB.DD. objeto-relacional PostgreSQL que permite almacenar IG en la propia base de datos. PostGIS incluye soporte para los índices espaciales R-Tree basados en SIGT y funciones para el análisis y procesamiento de objetos SIG y, a partir de su versión 2.0 (2008), comienza a dar soporte a almacenamiento de elementos 3D y funcionalidades relacionadas con estos elementos (Figura 2).

La fundación OSGeo es una organización no gubernamental que trata fundamentalmente de promover tecnologías para el tratamiento de información geoespacial y datos abiertos. Fue constituida en febrero de 2006 para proporcionar apoyo financiero, legal y organizativo a toda la enorme comunidad geoespacial de software libre y software de código abierto. 


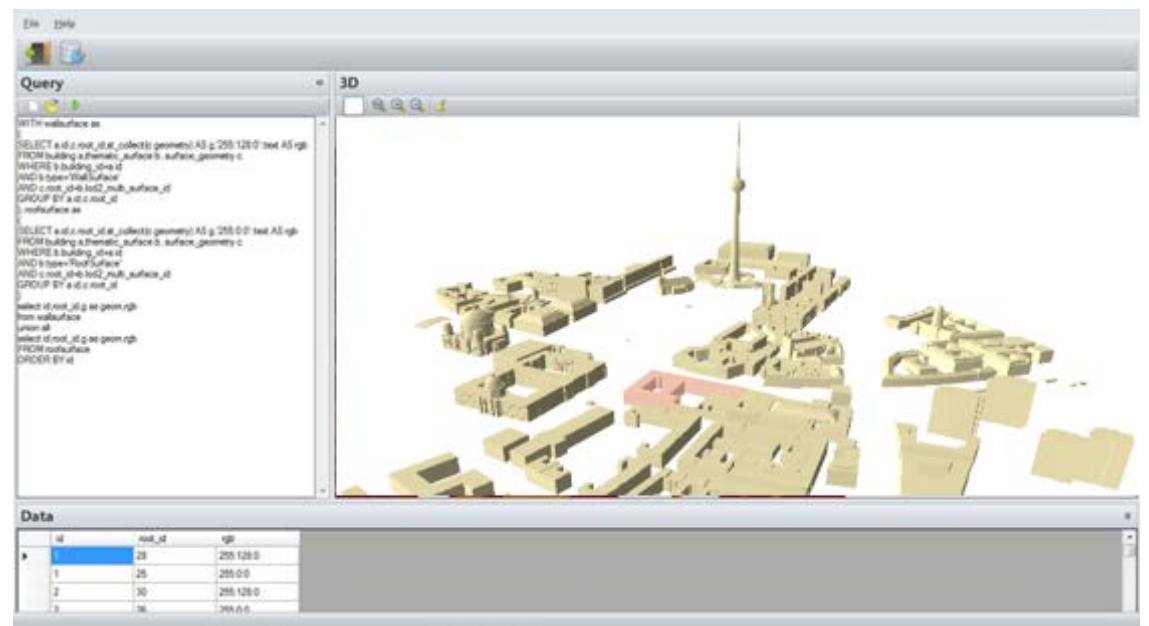

Figura 2. Visor 3D de PostGIS. Elaboración propia.

La fundación OSGeo es una organización no gubernamental que trata fundamentalmente de promover tecnologías para el tratamiento de información geoespacial y datos abiertos. Fue constituida en febrero de 2006 para proporcionar apoyo financiero, legal y organizativo a toda la enorme comunidad geoespacial de software libre y software de código abierto.

Finalmente, relacionado con las BB.DD. y la IG tridimensional, merece mención el trabajo desarrollado por el Departamento de Geoinformática de la Universidad Técnica de Munich, con su desarrollo de 3D City DB. Se trata de una plataforma de base de datos geográfica, abierta, que almacena, representa y administra modelos de ciudades 3D virtuales sobre una base de datos relacional espacial estándar. En este caso, el estándar utilizado es CityGML, con posibilidad de trabajar objetos urbanos semánticos y multidetalle. Dispone, además, de herramientas para facilitar el intercambio de datos como los exportadores a formato KML, COLLADA y glTF para la visualización en plataformas como Google Earth, ArcGIS y Cesium, basado en WebGL, y del que se hablará en profundidad en posteriores capítulos por la importancia que está adquiriendo.

Estos tres últimos hitos suponen un avance importante hacia el desarrollo futuro de IDE 3D, ya que permiten abordar el almacenamiento, gestión y análisis de elementos geométricos tridimensionales dentro de una misma BB.DD. Facilitando así la labor de gestión centralizada de la IG en una única plataforma.

En la segunda década del siglo XXI quizá las aportaciones más interesantes han venido del citado OGC. Desde 1994, el OGC ha estado reuniendo diversas compañías, grupos de investigación y organizaciones gubernamentales para 
desarrollar estándares de interfaz y codificación que solucionen problemas de la industria relacionados con el intercambio de IG 3D. Y como el propio grupo de trabajo describe, su alcance se está expandiendo debido, fundamentalmente a nuevas iniciativas que se ocupan de la ubicación de los nuevos desarrollos tecnológicos, tales como Internet de las cosas (IoT), la comunicación de máquina a máquina (M2M), imágenes ópticas, Realidad Aumentada (AR), navegación en interiores, dispositivos móviles incorporados, las redes sociales.

Según el propio OGC, estos nuevos desarrollos tecnológicos impactarán directamente sobre distintas áreas técnico-científicas de la arquitectura, ingeniería, construcción, etc. Nuevas industrias, como el marketing basado en la localización están creando demanda de datos y nuevas fuentes de datos sobre edificios, áreas comerciales e infraestructuras. Por este motivo, el OGC adoptó en 2016 IndoorGML, lenguaje del que se hablará en posteriores capítulos, como estándar para la localización y navegación en interiores.

La interoperabilidad de la ubicación 3D real, en las citadas áreas técnicocientíficas, está requiriendo la integración de la información de la ubicación, no solo tridimensional, también interior y exterior (con las implicaciones que esto implica de información tridimensional). Si a esto añadimos los grandes esfuerzos que desde las compañías de CAD, SIG y BIM se están desarrollando en ofrecer plataformas que gestionen la IG tridimensional (Valencia, J. et al., 2015), con sus correspondientes funcionalidades de importación/exportación a lenguajes estandarizados, como CityGML, nos encontramos en una situación propicia para afrontar el desarrollo de IDE 3D.

\section{Fortalezas y debilidades de las IDE 3D}

En el momento actual las IDE poseen el potencial y los medios suficientes para poder gestionar y publicar información tridimensional, si bien existen algunas lagunas en determinados servicios o componentes de las mismas.

En lo referente a la parte correspondiente a la gestión interna de una IDE, existen las suficientes herramientas, modelos, formatos, etc., para poder ser desarrolladas. Se anota la situación de continuo avance tanto en el desarrollo de herramientas de gestión, con potentes programas y BB.DD., así como las variadas líneas de investigación en lo referente a algorítmica para la creación de información semántica a partir de grandes nubes de puntos. Se están haciendo continuos avances para obtener información semántica a partir de grandes nubes de puntos y los sistemas de gestión de este tipo de información se manejan sin problemas desde sistemas comerciales desarrollados para visualizaciones realistas hasta sistemas de gestión de BB.DD. para manejos y accesos rápidos a las mismas.

En este punto también es necesario citar aquellas ramas tecnológicas que son capaces de aportar directamente modelos 3D, perfectamente elaborados desde el 
punto de vista semántico, como el CAD 3D y, sobre todo los BIM. En este sentido, ambas tecnologías son capaces de generar modelos 3D semánticos con la suficiente precisión como para ser visualizados a un LoD 4 (El-Mekawy, M., 2010).

En esta interesante sinergia entre los BIM y las IDE podemos diferenciar dos tipos distintos de modelos tridimensionales de ciudad, los modelos de diseño y los modelos del mundo real. Los primeros se utilizan generalmente para los propósitos de la industria de la construcción y para cumplir con los requisitos de un nivel máximo de detalle en la industria de la arquitectura, la ingeniería y la construcción. Los modelos del mundo real, sin embargo, son sistemas de información geoespacial que representan objetos espaciales ampliamente representados en aplicaciones SIG. Los esfuerzos de investigación en la industria de la construcción dieron como resultado los BIM, modelos que soportan la gestión de la información a lo largo del ciclo de vida de los edificios.

Los resultados de diferentes esfuerzos de integración de BIM e IDE muestran que sólo la información geométrica en 3D no cumple el propósito de integración y puede conducir a la inconsistencia geométrica. Se requiere más información semántica compleja (Figura 3).
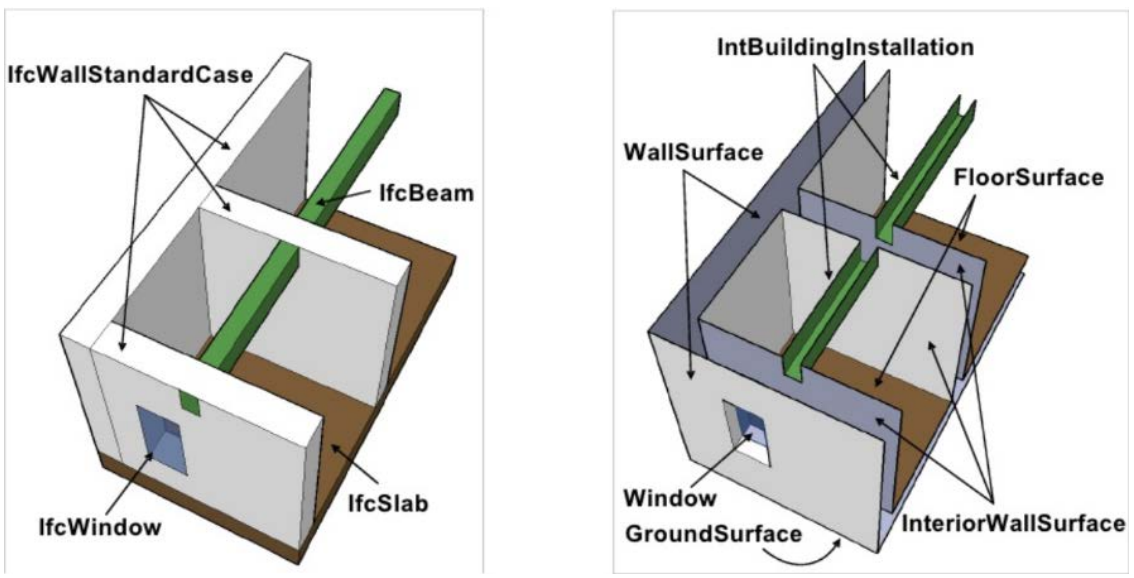

Figura 3. Modelo BIM, IFC (izquierda). Modelo IDE, CityGML (derecha) (Thomas H. Kolbe).

En lo referente a las normas de metadatos aplicables a las IDE, se observan lagunas en lo que a la IG tridimensional se refiere. Dichas lagunas se comunican, como no podía ser de otra forma, a los servicios de catálogo, encargados de permitir las búsquedas y filtrados de IG tridimensional. Pero también se vislumbran soluciones a estas carencias en futuras revisiones de las distintas normas. 
La normativa que mejor acogida tiene con la información tridimensional es la ISO 19115, contando con una serie de atributos básicos. Sin embargo, hay una serie de problemáticas asociadas a la misma respecto a las necesidades de desarrollo de una IDE 3D (Dietze, L. et al., 2007). En el caso de todos aquellos modelos basados en LoD, debería existir la posibilidad de ampliar aún más la ISO 19115 en este sentido. Tengamos en cuenta que el LoD, por ejemplo en los modelos CityGML va implícito en el archivo, sin embargo con la intención de facilitar los servicios de búsqueda y de catálogo, esta información debería estar disponible en los metadatos. Una posibilidad para solucionar este problema sería la subclase MD_DataIdentification de la clase MD_Identification, con un nuevo atributo levelOfDetail, con valor entero entre 0 y 4 para definir el LoD. Por supuesto, esta problemática se repite en la norma Dublin Core.

Otro problema adicional es que en las normas de metadatos analizadas no se permite conocer con mayor precisión qué información de partida se utilizó para la creación de los modelos 3D. Aunque la ISO 19115 permite definir la calidad de los MDE, no es capaz de profundizar en este dato.

Finalmente, otro problema detectado es la escasa capacidad que tienen las normas para informar sobre los elementos semánticos de un modelo 3D. Si bien muchos modelos tridimensionales incorporan una extensa información semántica sobre los elementos que incorpora, sobre todo los modelos de ciudad con un LoD 3 o 4, las normas de metadatos analizadas hasta el momento no son capaces de recoger e informar sobre esta riqueza de información semántica.

De todo lo analizado hasta el momento con respecto a los metadatos y las IDE 3D, se puede concluir que aunque existe una mínima estructura capaz de soportar este tipo de información, no han sido todavía desarrolladas para ofrecer todo el potencial de un esquema de metadatos a nivel de IG tridimensional. Sin embargo, existen posibilidades de ampliación gracias a las figuras de propuestas de desarrollo de las distintas normativas, por lo que se puede concluir que, aunque no están totalmente desarrolladas estas normativas, conforme se vayan presentando y aprobando dichas propuestas, el grado de desarrollo provocará que a corto plazo, dichas normativas sean capaces de afrontar el desarrollo de IDE 3D.

Por lo que respecta a los protocolos de intercambio y comunicación, se constata la existencia de suficientes servicios diseñados y desarrollados para servir modelos 3D. Dichos modelos pueden ser tanto continuos como discretos, WCS y WFS, llegando a cubrir con ellos todos los LoD definidos por el OGC. Sin embargo, se han detectado algunas lagunas importantes, sobre todo en lo referente al lenguaje de intercambio en los servicios WFS, mostrando las debilidades y los puntos fuertes de los lenguajes de intercambio, como CityGML y resaltando la existencia de formatos más eficientes en materia de intercambio de modelos 3D, como JSON. A pesar de ello, las nuevas 
mejoras que se implementen en futuras versiones de CityGML, junto con la adopción de nuevos estándares por parte del OGC y las conexiones existentes entre este lenguaje y otros con gran potencial práctico, como IndoorGML hacen pensar que CityGML será uno de los principales medios de comunicación de modelos 3D.

Finalmente, en lo que respecta a la parte del cliente final de una IDE, existen distintas soluciones para poder llevar a cabo los procesos de visualización, consulta, navegación, etc. De las dos grandes posibilidades de publicación de IG tridimensional, mediante clientes pesados o mediante clientes ligeros, existen soluciones para poder llevar a cabo este proceso, bien directamente a través de la carga de ficheros mediante archivos, bien mediante conexiones del tipo WFS.

Además se ha podido comprobar la gran cantidad de investigaciones que se están desarrollado en los últimos años sobre la publicación de IG 3D, poniendo el foco de atención en la publicación por Internet. A pesar de esto, es necesario poner de manifiesto la necesidad de herramientas capaces de aglutinar tanto las posibilidades de conexión a modelos 3D vía fichero (CityGML, kml, etc.) o vía conexión WFS, manteniendo las capacidades de análisis y procesado avanzados de modelos 3D, en un entorno de navegación ligero, intuitivo y fácilmente interaccionable. Es decir, se trataría de herramientas con la comodidad de uso en lo relativo a interface, que se puede ver en los clientes ligeros, añadiendo la potencia de análisis y gestión de los clientes ligeros, tanto a nivel de procesado como a nivel de creación de elementos 3D.

\section{Modelo funcional propuesto de una IDE 3D}

Para finalizar, con la intención de completar el ciclo investigador, se propone el siguiente esquema funcional, señalando las deficiencias o carencias encontradas y proponiendo cambios o medidas a tomar para hacer posible el desarrollo de las IDE 3D. Dicho diseño se muestra en las tres siguientes imágenes (Figuras 4, 5 y 6).

Se constata la situación de continuo avance tanto en el desarrollo de herramientas de gestión, con potentes programas y BB.DD., como en las variadas líneas de investigación que abordan lo referente a algorítmica para la creación de información semántica a partir de grandes nubes de puntos. Una vez creados los modelos, tenemos la posibilidad de afrontar el desarrollo tanto desde un punto de vista de sistema libre, como utilizando sistemas propietarios, si bien la filosofía intrínseca de una IDE es la del desarrollo lo más abierto posible.

Tras esta distinción, el siguiente paso sería el desarrollo del back-end, habiendo cumplido previamente las distintas normativas (INSPIRE) en la parte referente al modelo de datos. En lo relativo tanto a la parte del back-end como del middleware (conjunto de componentes desarrollados que sirven para integrar aplicaciones) (Figura 5), en este apartado sería necesario contemplar la normativa en materia de metadatos aplicables a las IDE. 


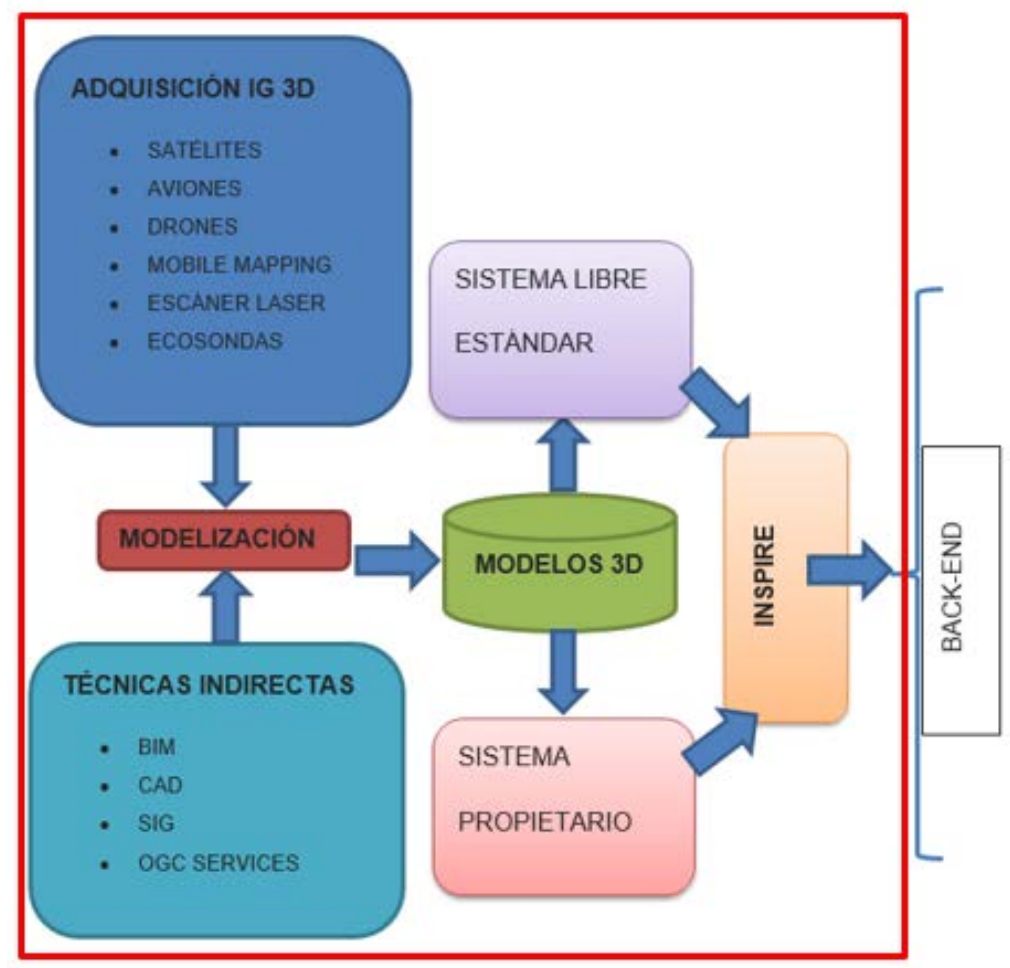

Figura 4. Elementos de adquisición de IG 3D para entrada en la IDE. Elaboración propia.

Respecto al sistema de almacenaje de los modelos 3D, tenemos distintas posibilidades, pasando desde un sencillo sistema de archivos, una BB.DD. relacional o, incluso incorporando las últimas tendencias en materia de BB.DD. no relacionales que están aportando importantes avances en la comunicación de grandes cantidades de datos (Big Data) de todo tipo, incluidos los geográficos. El paso del back-end al middleware se podría hacer en función de la alternativa de almacenaje, mediante archivos (CityGML, JSON, etc.) o sentencias SQL, para las BB.DD. relacionales.

Por lo que respecta a los protocolos de intercambio y comunicación, la parte del middleware, a lo largo de la presente investigación se ha constatado la existencia de suficientes servicios diseñados y desarrollados para servir modelos 3D. Todo esto, junto con las distintas tipologías de lenguajes y formatos de intercambio de modelos 3D, nos daría paso a la parte de front-end, bien mediante servicios HTTP, FTP o web services. 


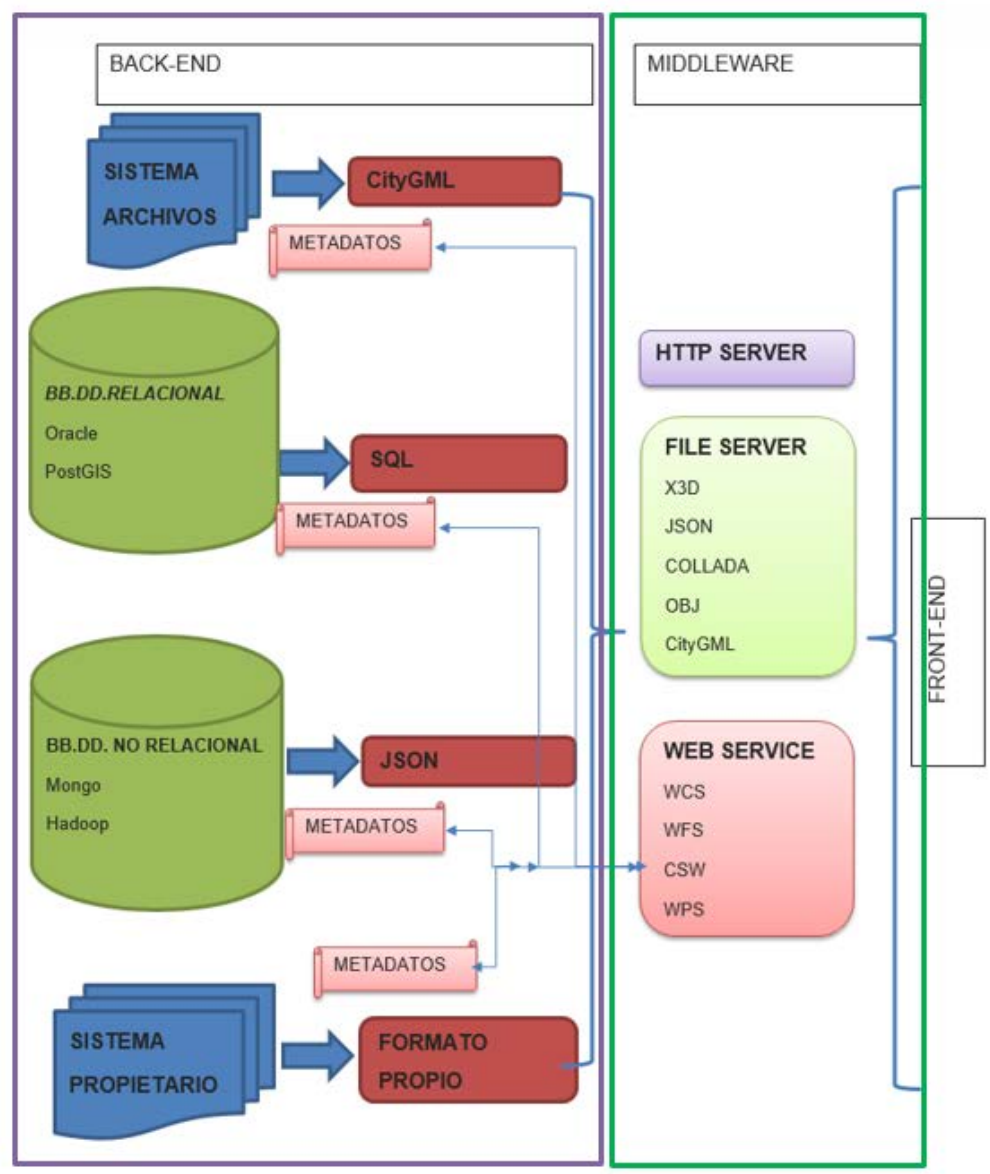

Figura 5. Esquema de Back-End y Middleware de una hipotética IDE 3D. Elaboración propia.

Finalmente, en lo que respecta a la parte del cliente final, la parte correspondiente al front-end, se propone una variante, dependiendo de si el uso lo va a hacer el usuario desde un navegador de internet (cliente ligero) o desde un software de escritorio (cliente pesado). Si la idea es el uso desde la primera opción se propone el desarrollo de aplicaciones web o la adaptación de globos con conexiones a los servidores que alojan la información tridimensional. Si, por el contrario, el usuario necesita más capacidad de análisis y geoproceso, se recomienda el uso de herramientas de escritorio que permitan la carga de modelos $3 \mathrm{D}$ y conexiones a servicios WFS. 


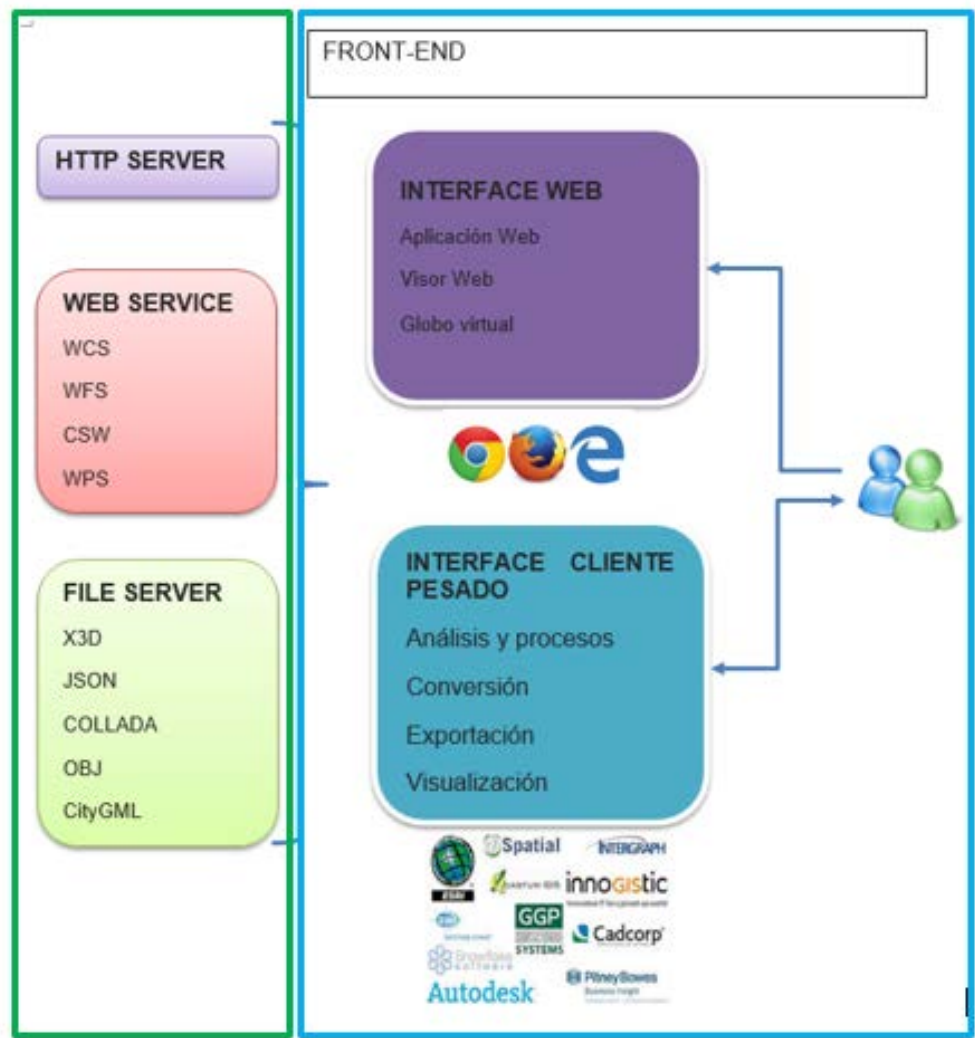

Figura 6. Esquema de Front-End de una hipotética IDE 3D. Elaboración propia.

\section{Las IDE 3D. Líneas futuras de investigación}

Son numerosas las líneas de investigación que se están desarrollando en relación con la IG tridimensional, sin embargo, de cara al desarrollo de una IDE, las líneas más interesantes a futuro serían:

\section{Desarrollo de BB.DD. con capacidad de almacenaje de modelos 3D}

La principal línea de investigación en lo que a la gestión de la información interna se refiere, es, sin duda, el desarrollo de BB.DD. con capacidad de almacenaje de modelos 3D. En este sentido tanto Oracle, como PostGIS y otras iniciativas menores están desarrollando importantes esfuerzos en mejorar y ampliar las posibilidades de gestión de modelos 3D. Con especial mención a los Gestores de BB.DD. NoSQL. 


\section{Algoritmos para obtención de modelos $3 D$ semánticos}

Otra línea muy prometedora es la del desarrollo de algoritmos para pasar de forma más o menos automatizada, de modelos 3D brutos a modelos 3D semánticos, tanto en lo que se refiere a nubes de puntos como a píxeles.

\section{Ampliación de normativa de metadatos en materia de IG 3D}

En lo que a los metadatos se refiere, las investigaciones a futuro se centrarán, sin duda, en la presentación de nuevas alternativas de ampliación de las actuales normas en materia de modelos 3D. A lo largo de esta investigación se ha mostrado la debilidad de éstas con respecto a la IG 3D, por lo que se hace del todo necesario desarrollar nuevas líneas de investigación de cara a resolver estos problemas.

\section{Mejora de los lenguajes de intercambio}

Probablemente la línea de investigación más importante en esta materia esté relacionada con la mejora del lenguaje de intercambio, ya sea definiendo nuevos protocolos, o mejorando los actuales (kml, GML). La posibilidad de dotar a los formatos de la propiedad del multidetalle o la mejora del modelo de CityGML para aumentar en versatilidad, son algunos ejemplos de posibles líneas de investigación.

\section{Publicación de IG 3D}

En lo que a las líneas de investigación a futuro relacionadas con las IDE 3D en materia de publicación, probablemente sea aquí dónde haya más recorrido. Existen numerosas iniciativas de cara a la publicación de modelos 3D en Internet, tanto en la parte de cliente, como en la parte de servidor. Además hay que destacar también las investigaciones que están realizando las compañías de desarrollo de software para crear plataformas de trabajo ágiles y potentes, tanto en lo respectivo a la navegación como en la parte de geoprocesos 3D.

\section{Conclusiones}

Del análisis de los anteriores apartados se puede finalizar con las siguientes conclusiones:

1. La tercera dimensión es realmente uno de los problemas a afrontar dentro de las IDE. Conforme a las investigaciones desarrolladas ha quedado constatada y verificada esta hipótesis, tal y como se desprende de entrevistas realizadas a importantes personas de renombre nacional e internacional en el área de las IDE. Por otro lado, también se puede inferir directamente la verificación de esta hipótesis del análisis del sistema de seguimiento y actualización de la investigación que se ha desarrollado para esta investigación, en el que se consi- 
gue rastrear más de 1400 enlaces bibliográficos en cinco años, relacionados directamente con palabras clave en materia de IDE 3D. La comunidad científica está desarrollando numerosas investigaciones en esta materia.

2. Las IDE 3D son realmente necesarias para dar solución a una serie de problemas que se plantean a corto/medio plazo en relación con distintas áreas técnico-científicas. La verificación de esta hipótesis llega del análisis de la bibliografía utilizada para esta investigación, en la que como ya se ha puesto de relieve, el medio más homogéneo, interoperable y estandarizado para la gestión y publicación de IG, incluyendo la tridimensional, son las IDE. Además se ha constatado que si a la potencialidad de una IDE le añadimos las posibilidades que los modelos tridimensionales ofrecen en materia de análisis en multitud de áreas técnico-científicas, el resultado final es una herramienta con mayor capacidad de obtención de respuestas y alternativas a la hora de la toma de decisiones relacionadas con el entorno y aplicable a una gran cantidad de áreas de estudio. Esta necesidad es también fácilmente extraíble del análisis de varias líneas de investigación que se han aportado en esta tesis, en las que se plasma el interés de comunicar plataformas técnico científicas, como los BIM, la realidad virtual, la realidad aumentada, por ejemplo, con las IDE tridimensionales.

3. Aunque la evolución de una manera estandarizada y rápida no es sencilla, las IDE 3D son proyectos abordables gracias a los distintos desarrollos e investigaciones que se han abordado en los últimos años respecto a la tercera dimensión y la IG. Del mismo modo se han mostrado lagunas o deficiencias y se han puesto en valor líneas de investigación dirigidas a hacer de las IDE 3D plataformas estandarizadas efectivas y eficientes de cara a la gestión y publicación de IG tridimensional. Por todo lo anterior, se puede decir que hay mucho trabajo desarrollado y que la hipótesis quedaría verificada, si bien tal y como en la misma se argumenta, quedan numerosas líneas por desarrollar y hacer llegar a los distintos protagonistas de una IDE, desde los desarrolladores hasta el usuario final.

4. Uno de los aspectos más importantes a afrontar por las IDE, relacionado con la tridimensionalidad, es la comunicación de la IG tridimensional a través de Internet. Conforme a la tendencia natural de las plataformas de uso de IG, en general, la progresión es ir avanzando en la publicación en Internet. Esto es totalmente aplicable a las IDE 3D, lo que se traduce en muchas de las líneas de investigación y aportes que se han presentado a lo largo de este trabajo. Conforme se van haciendo progresos en materia de publicación de modelos 3D en Internet, sobre todo enfocados a juegos y plataformas de ocio, las IDE se nutren de estos aportes para realizar progresos en paralelo, como no podía ser de otra forma. 


\section{Bibliografía}

Ahearn, L., (2005). "El arte de los juegos 3d/the art of 3d games (diseño y creatividad)”, Anaya Publishers.

Basanow, J.; Neis, P.; Neubauer, S.; Schilling, A. and Zipf, A., (2008). “Towards 3D spatial data infrastructures (3D-SDI) based on open standards - experiences, results and future issues”, Advances in 3D geoinformation systems, Springer, pp. 65-86.

Dietze, L.; Nonn, U. and Zipf, A., (2007). "Metadata for 3D city models analysis of the applicability of the ISO 19115 standard and possibilities for further amendments", The 10th AGILE International Conference on Geographic Information Science, pp. 1-9.

El-Mekawy, M., (2010). Integrating BIM and GIS for 3D City Modelling: The Case of IFC and CityGML.

Jiménez Macías, E.; Pérez de la Parte, Mercedes; Martínez Cámara, E.; Sanz Adán, F.; Santamaría Peña, J. y Blanco Fernández. J.Escenarios virtuales WEB3D: simulación con VRML, JAVA3D y X3D.

Lafont Morgado, P., (1999). “Prototipado rápido e ingeniería inversa”, Revista de Plásticos Modernos, 77 (514), pp. 384-390.

Montilla, G.; Bosnjak, A. y Villegas, H., (2002). "Visualización de mundos virtuales en la medicina. Bioingeniería En Iberoamérica: Avances y Desarrollos”, Cap. XX, Carmen Muller-Karger, Miguel Cerrolaza (eds.).

Scianna, A. and Ammoscato, A., (2010). "3D GIS data model using open source software. Core Spatial Databases-Updating, Maintenance and Services-from Theory to Practice”, Haifa, Israel, 15-17 March 2010.International Archives of Photogrammetry, Remote Sensing and Spatial Information Sciences, 38 (Part 4), 8-2.

Van Oosterom; Petrus Johannes Maria; Zlatanova, S. and Penninga, F., (2008). Advances in 3D geoinformation systems, Springer.

Valencia, J.; Muñoz-Nieto, A. and Rodríguez-Gonzálvez, P., (2015). "Virtual modeling for cities of the future”, isprs - International Archives of the Photogrammetry, Remote Sensing and Spatial Information Sciences, XL-5/W4, pp. 179185.

Varela García, F.A.; Hernández Ibáñez, L.A.; Taibo Pena, J. y Seoane, A., (2007). "Ampliación de las capacidades de visualización de un SIG libre mediante la comunicación con un navegador 3D”. 